\title{
Interconnect Design Methods for Memory Design*
}

\author{
Chanseok Hwang \\ Department of Electrical Engineering-Systems \\ Univ. of Southern California, Los Angeles, CA 90089, USA \\ Tel : 1-213-740-4472 Fax : 1-213-740-9803 \\ chanseoh@usc.edu
}

\begin{abstract}
This paper presents a solution to the problem of designing interconnects for memory devices. More precisely, it solves the automatic routing problem of memory peripheral circuits as an over-the-cell channel routing problem under pre-specified routing topologies and performance constraints. The proposed routing method, named TANAR, consists of two steps: a performance-driven net partitioning step, which constructs a routing topology for each net according to performance constraints, and a performance-driven track assignment step, which reduces the crosstalk noise. Experimental results demonstrate that TANAR significantly reduces both crosstalk for noise sensitive nets, and delay for timing critical nets while minimizing channel height.
\end{abstract}

\section{INTRODUCTION}

Memory devices are perhaps the most common components on integrated circuits. They are generally classified into DRAM, SRAM, Mask ROM and Flash Memory according to how they store data. Memory devices comprise of a memory arrays and peripheral circuits. The memory arrays can be designed manually because of its highly regular layout structure. In contrast, the peripheral circuits pose a significant challenge to memory designers. Figure 1 shows the typical architecture of a 1GB DRAM. Although area of the peripheral circuits is only about $20 \%$ of the whole chip area, it normally takes much longer to design the peripheral circuits compared to the memory array. This is because layout designers must carefully design the peripheral circuits to avoid crosstalk noise and meet critical path delay, often by exploiting a full-custom design flow. In addition, they must pay close attention to layout area minimization, which is quite important especially for mass-produced integrated circuits such as the memory devices.

We next describe a number of key facts related to the design of peripheral circuits for memory devices. Address and data signals should be routed with the shortest connections and be immune from crosstalk noise. Analog signals used for voltage converter circuitry should be shielded from crosstalk noise. Over-the-cell area should be effectively exploited in order to reduce the overall chip area. Notice that in these peripheral circuits, the size of transistors is large in order to provide a high-current drive strength [1]. In practice, the logic cell height in peripheral circuits tends to be about 100um whereas the standard cell height in ASIC designs is about 10um. Finally, the process technology used to fabricate memory devices (especially

* This research was supported in part by NSF under grant no. 9988441.

\author{
Massoud Pedram \\ Department of Electrical Engineering-Systems \\ Univ. of Southern California, Los Angeles, CA 90089, USA \\ Tel : 1-213-740-4458 Fax : 1-213-740-9803 \\ pedram@usc.edu
}

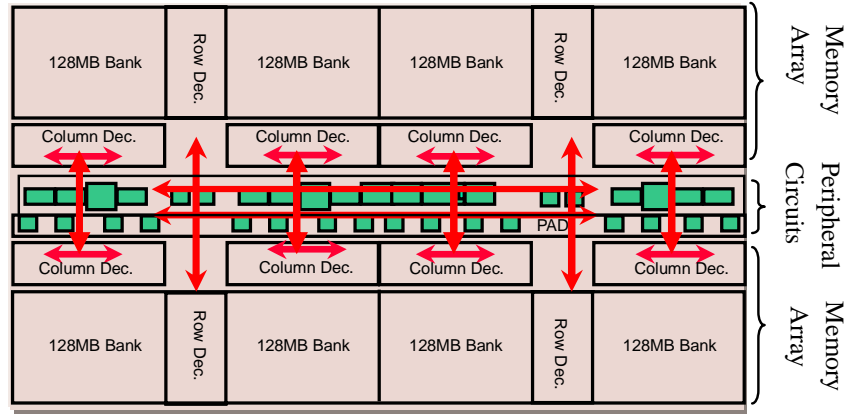

Figure 1. An example of 1GB DRAM architecture

for DRAM devices) provides only two or three metal layers in order to reduce production cost.

In this paper, we solve the automatic routing problem of peripheral circuits with performance constraints as a performance-driven over-the-cell channel routing problem. The reasons for this approach are:

1.The crosstalk problem can be solved more easily in channel routing compared to area routing. This is because of the fact that wire segments in channel routing are processed track-by-track. This in turn allows us to do an efficient and accurate analysis of crosstalk correlations between neighboring nets.

2.The layout shape of a typical peripheral circuit is a long rectangle, which makes it more amenable to channel-based routing.

3. Memory devices use only two or three metal layers (except for high-speed SRAM devices which tend to use five or more metal layers). This makes channel routing an especially effective technique for routing the memory devices. Notice that channel routing is not normally used for multi-layer (more than 3-layers) routing problems, because its routing efficiency tends to be lower than that of area routing. However, for two or three layers routing problem, channel routing algorithms [2-4] have an edge over the area routing.

The remainder of the paper is organized as follows. Section 2 introduces the models of fixed net topology, timing, crosstalk noise, and cell layout style used in this paper. The proposed routing algorithm and some extensions are described in section 3 and 4, respectively. Experimental results are shown in Sections 5. We shall draw concluding remarks in Section 6. 


\section{PRELIMINARIES}

\subsection{Fixed Topology Model}

The peripheral circuits include nets with various performance constraints. To simultaneously meet these constraints and achieve our design goals, we introduce the notion of a fixed topology net, that is, a net with the predefined routing topology. In particular, we define four types of fixed routing topologies according to performance constraints. First, we classify signal nets in peripheral circuits into four types:

1. Delay critical and crosstalk noise sensitive nets (called critical nets and denoted by $N_{C}$ ): Signal nets that belong to address and data signal nets.

2. Crosstalk noise sensitive nets (called sensitive nets and denoted by $N_{S}$ ): Signal nets that reside in the circuitry of pre-charge, domino logic or are used to carry low voltage swing signals (especially for analog nets).

3. Delay critical nets (called timing nets and denoted by $N_{T}$ ): Signal nets that lie on the timing-critical paths.

4. Non-critical digital nets (called base nets and denoted by $N_{B}$ ): Signal nets that have sufficiently positive noise margin and timing slack so that the interconnect topology in channel routing for the nets does not affect the circuit performance.

Each type of nets has its corresponding routing topology. Figure 2 shows four types of routing topology with source-to-sink delay for each sink, and table 1 summarizes critical sink delay and consumed channel area for each of these routing topologies. Delay is calculated by using the Elmore delay [5] model to be reviewed in Section 2.2. For simplicity, we assume unit capacitance and resistance per unit length. The grid marks in this figure represent the unit length. The source has a drive resistance of 5 units, and all sinks have a load capacitance of 1 unit. The entries in the second row of table 1 correspond to the critical sink delay from a source, and the amount of delay increase compared to topology III.

Note that the routing results significantly vary depending on the routing topology that is being used. Topology-I for $N_{C}$ has the single track from which branches connect to the pins. This topology improves the signal propagation and routability [6] because the bus line is routed with the shortest straight line and all the sinks have rather similar delays from the source. To minimize crosstalk noise, the routing topology-II, which results in the minimum total routing length, is desirable. This is because the coupling capacitance that causes crosstalk is proportional to the total coupling length, which will in turn be shorter if the total routing length is shorter. The delay between a source and a specific critical sink of a multi-terminal net is minimized via topology-III. Critical paths in peripheral circuits are usually well defined. Therefore, minimizing the critical sink delay is more effective in reducing the critical path delay than minimizing the maximum delay of a net.

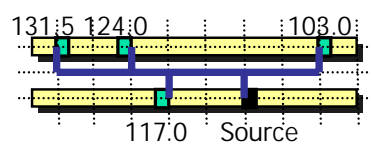

(a) Topology-I for $N_{C}$

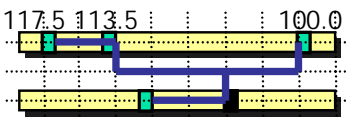

84.0(CS) Source

(c) Topology-III for $N_{T}$

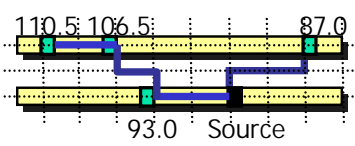

(b) Topology-II for $N_{S}$

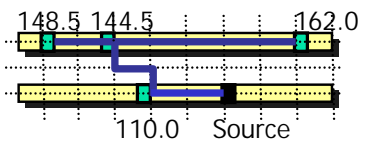

(d) Topology-IV for $N_{B}$
Figure 2. Routing solutions corresponding to different topologies for a net comprised of a source and four sinks.

TABLE 1. Comparisons of routing performances for four routing topologies.

\begin{tabular}{|c|c|c|c|c|}
\hline Topology type & I & II & III & IV \\
\hline Critical sink delay & $117(39 \%)$ & $93(10 \%)$ & 84 & $110(30 \%)$ \\
\hline Total routing length & 12 & 11 & 12 & 12 \\
\hline $\begin{array}{c}\text { Consumed } \\
\text { channel area }\end{array}$ & 12 & 7 & 8 & 3 \\
\hline
\end{tabular}

Topology-IV can significantly reduce channel height for a circuit with large cell heights and many base nets, which is the case for peripheral circuits of memory devices. Note that although the total routing length is nearly the same for the four topologies, the delay and routing length used inside the channel are quite different. In this paper, we use these observations by enforcing the notion of fixed net topologies, that is, each net has its optimal routing topology according to its performance constraint.

\subsection{Delay Model}

When computing interconnect delay, we use the Elmore delay [5]. Thus we briefly review this model here.

Elmore Delay Calculation: Consider a routing tree $T$ for net $n_{i}$. Each edge $e_{v}$ of the tree is modeled as a $\pi$-type RC circuit comprised of a resistor $r_{e v}$ and two capacitors $c_{e v} / 2$, where $r_{e v}$ is the total wire resistance and $c_{e v}$ is the total capacitance of edge $e_{v}$. The Elmore delay from source $p_{0}$ to sink $p_{i}$ is calculated by:

$$
\begin{gathered}
D\left(p_{0}, p_{i}\right)=R_{d} C_{p 0}+\sum r_{e v}\left(c_{e v} / 2+C_{v}\right) \\
\mathrm{e}_{\mathrm{v}} \in \operatorname{path}\left(p_{0}, p_{i}\right)
\end{gathered}
$$

where $R_{d}$ is the output drive resistance at the source of $T$ and $C_{v}$ is the total sub-tree capacitance at $T_{v}$.

\subsection{Crosstalk Model}

When designing crosstalk noise immune interconnection, we use the peak crosstalk noise amplitude equation proposed in [7]. This equation shows high accuracy and simplicity for its application to layout techniques like routing. For a victim RC tree coupled to an aggressor, the peak noise at any node is calculated by: 


$$
P N=\frac{\sum_{R_{i} \in \Omega} C_{X i} R_{i}}{\sum_{C \in \Lambda} C_{i} R_{i i}}
$$

where $C x_{i}$ is the sum of downstream coupling capacitances seen from node $i, \Omega$ is the union of the victim driver resistance and the set of resistances in the unique path from the root to the node, $\Lambda$ is the set of all capacitors, and $R_{i i}$ is the resistance seen across $C_{i}$ with all capacitors open circuited (refer to [7] for details). Figure 3 shows two on-chip lines running parallel on the same metal layer and its equivalent circuit. We assume line 2 is quiescent (as a victim net) when line 1 is switches (as an aggressor net). The peak noise voltage on line 2 caused by crosstalk between two lines is expressed by using the equation (2). The lumped $\pi$ model is used to model the interconnection. $C x$ denotes half of the total coupling capacitance. $C 1$ and $C 2$ are half of the line capacitances whereas $C 3$ and $C 4$ denote sums of the respective half line capacitances and receiver capacitances. $R l$ is the aggressor driver resistance, $R 2$ is the victim output resistance, and $R$ is the line resistance [7].

Switching window is in general one of the key factors when crosstalk noise effects are considered. In memory circuit design, however, noise sensitive nets are mostly analog signals such as reference voltage signals with small voltage swing. As a result, we may assume that all noise sensitive nets must be kept immune to crosstalk noise at all times.

\subsection{Cell Model}

It is necessary to efficiently exploit over-the-cell area and thereby minimize channel height. This is especially important for peripheral circuits of the memory devices, which have large cell height. The efficiency of routing algorithms is largely dependent on the physical constraints that exist over the cell. Figure 4 depicts the cell model of peripheral circuits. This is often called "target-based cell" and is carefully designed to allow effective utilization of over-the-cell area during channel routing. The model of Figure 4 is the same as that of the Target-Based Cell [9] except that here power and ground lines are implemented in M2 layer. Pins are in the form of long vertical strips in M1 layer. In addition, the cell is filled with M1 obstructions resulting from the internal wires, and dummy features that are added to achieve oxide planarization [10]. M2 can be therefore freely used over-the-cell area except where the power and ground lines are, whereas M1 is forbidden in the over-the-cell area except for where the pins are. In the case of using M3 layer, the cell model may be changed (described in Section 4).

\section{PROPOSED ROUTING ALGORITHM}

The automatic routing problem of peripheral circuits of memory devices can be defined as follows.

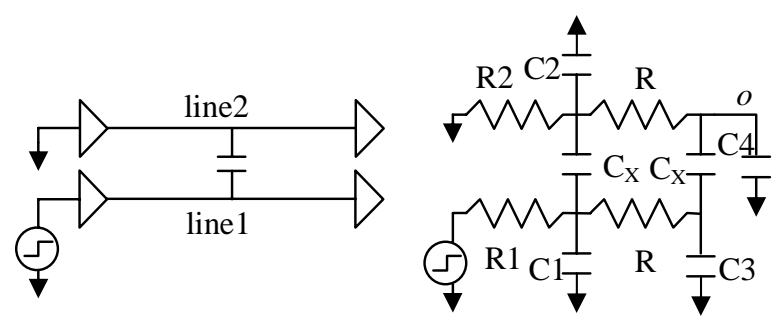

Figure 3. Noise coupling (a) Circuit configuration (b) Equivalent circuits; the peak noise is calculated by using (2) is as:

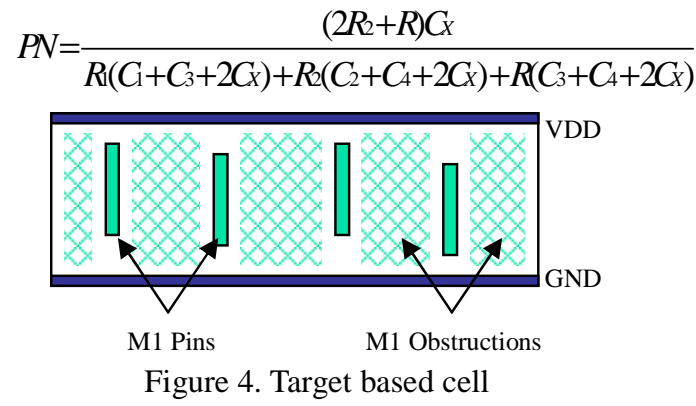

Problem Statement: Given a set of nets $N$ to be routed inside a channel using two or three routing layers, the delay and noise information, do detailed routing of $N$ such that the performance constraints are satisfied and channel height is optimized.

TANAR solves the given problem by applying two steps: a performance-driven net partitioning (PDNP) step, which decides a fixed topology for each net according to its net type and partitions the topology in order to build it during the routing, and a performance-driven track assignment (PDTA) step, which satisfies the delay and noise constraints while minimizing channel height.

\subsection{PDNP Problem and Algorithms}

The objective of the PDNP step is to construct an optimal routing topology for $N_{C}, N_{S}, N_{T}$ and $N_{B}$, respectively, and then partition a net into sub-nets to build it during channel routing. The four types of nets have different performance constraints; therefore, the routing topology of each type of net should be optimized so that it satisfies the corresponding constraint. We specify the optimal routing topology for each net type and algorithm used to build the topology next.

- BRT for $N_{C}$ : Given a net $n \in N_{C}$ with pins $p_{i}(i=1,2,3$, $\ldots)$ in a channel, the Bus Routing Tree $(B R T)$ is a steiner tree $T$ which consists of a single track and a number of branches at steiner points on the track connecting $p_{i}$ 's. BRT can be constructed by laying a single track from the left-most pin to the right-most pin of the net in a channel during PDTA and connecting pins to the track.

- MST for $N_{S}$ : Given a net $n \in N_{S}$ with pins $p_{i}(i=1,2,3$, ...) in a channel, the MST algorithm $\operatorname{MST}(n)$ finds a spanning tree $T$ which minimizes the total length of the $T$. 
This is because crosstalk noise is caused by the coupling capacitance, which is proportional to the coupling length; therefore, the routing topology for $N_{S}$ should be optimum in terms of total length. The well-known Prim's algorithm of [11] is used for $\operatorname{MST}(n)$.

- CSRT for $N_{T}$ : Given a net $n \in N_{T}$ with a source $p_{0}$, a critical sink $p_{c}$ and other sinks $p_{i}(i=1,2,3, \ldots)$ in a channel, the Critical Sink Routing Tree algorithm $\operatorname{CSRT}($ ) finds a spanning tree $T$ which minimizes the Elmore delay from $p_{0}$ to $p_{c}$ in the $T$. CSRT() originates from the ERT algorithm of [12] which generates a spanning tree such that the maximum Elmore delay from source to any sink is the minimum. CSRT() starts with a MST $\left(T-p_{c}\right)$ and then connects $p_{c}$ to $T$ so that adding edge $\left(p_{c}, p_{i}\right)$ or $\left(p_{c}, p_{0}\right)$ yields a tree with the minimum critical sink delay from the source.

- MCAT for $N_{B}$ : Given a net $n \in N_{B}$ with pins $p_{i}$ (i=1,2, $3, \ldots)$ in a channel, the Minimum Channel Area Tree algorithm $\operatorname{MCAT}(n)$ finds a spanning tree $T$ which consumes the minimum channel area. Figure 5 shows the process of constructing this tree. $\operatorname{MCAT}(n)$ works as follows. First, it adds edges between top-pins $P_{T}$ in the upper row, which forms the top-tree $T_{T}$ (see Figure 5(a)). Next it adds edges between bottom-pins $P_{B}$ in the lower row, which forms the bottom-tree $\mathrm{T}_{\mathrm{B}}$ (see Figure 5(b)). Finally, it finds a pair of pins $(u, v)$ with the minimum length, where $u \in T_{T}, v \in T_{B}$ (see Figure 5(c)).

Notice that BRT, CSRT, MST and MCAT correspond to topology-I, II, III and IV, respectively in Figure 2. In Figure 7, we illustrate the PDNP algorithm with an example. The algorithm first constructs $T_{M S T}, T_{C S R T}$, and $T_{M C A T}$ via MST(.), CSRT(.), and MCAT(.) for $N_{T}, N_{S}$, and $N_{B}$, respectively. We impose the upper bound on the increase in delay of the base nets. Therefore, the PDNP algorithm compares the delay of $T_{M C A T}$ with that of $T_{M S T}$. It will then use $T_{M C A T}$ for nets in $N_{B}$ only if the delay increase is equal to or less than some fixed threshold (say 30\%). Otherwise, it uses $T_{M S T}$ for nets in $N_{B}$. Next, it partitions $T$ into sub-trees $T_{s u b}$ in such a way that each edge $e$ of $T_{C S R T}$ and $T_{M S T}$ forms a sub-tree, resulting in a sub-net with two pins, and $T_{T}, T_{B}$ and $e_{v u}$ of $T_{M C A T}$ forms each their sub-tree, generating three sub-nets. These sub-nets are independently assigned to a specific track during the track assignment. Figure 6 shows the results of partitioning a tree into sub-trees for these three trees. Note that BRT is not partitioned into sub-trees such that the whole pins are connected to the single track.

\subsection{PDTA Problem and Algorithm}

The objective of the PDTA is to assign nets to tracks in a channel so as to minimize the channel height while meeting the horizontal constraints (HC), vertical constraints (VC) and noise constraints (NC).

HC: If two horizontal segments with overlapping spans belong to different nets, then they should not be assigned to the same track.

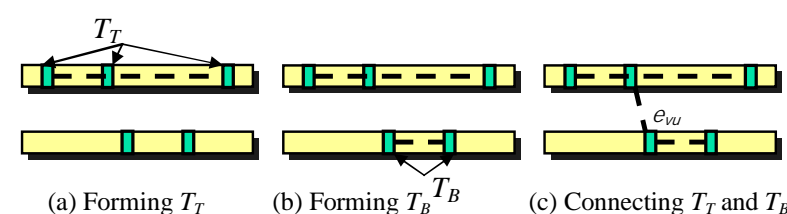

Figure 5. The process of constructing MCAT

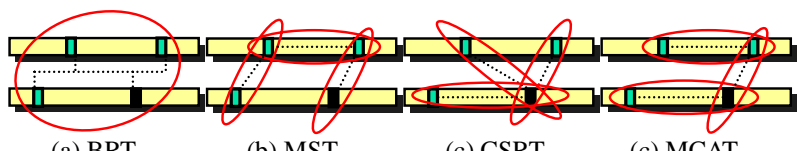

(a) BRT

(b) MST

(c) CSRT

(c) MCAT

Figure 6. The results of partitioning a tree into sub-trees for four different routing trees

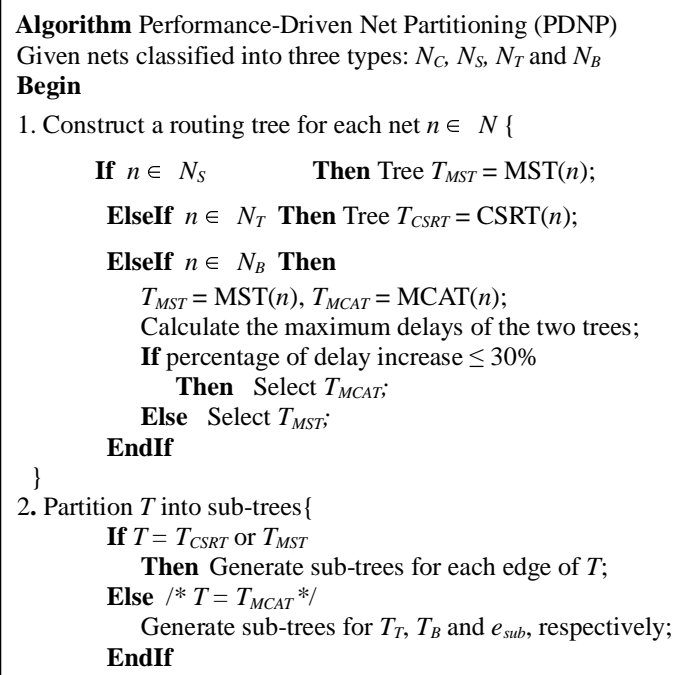

Figure 7. The performance-driven net partitioning algorithm

VC: When two pins belonging to different signal nets are in the same column, the track to which the upper pin in that column connects must be placed above the track to which the lower pin in that column connects.

NC: $P N(i) \leq A N(i)$ for a net $n_{i} \in\left\{N_{C}, N_{S}\right\}$ should be satisfied, where $P N(i)$ is peak crosstalk and $A N(i)$ is the maximum allowable peak crosstalk noise for a net $n_{i}$.

The PDTA algorithm proceeds track by track from the over-the-cell areas towards the middle of channel. Figure 8 shows the order of tracks used by this algorithm, which is based on the work by Yoeli in [3]. The rationale for this track ordering is that over-the-cell areas can be used to the highest degree (tracks of the over-the-cell areas in the upper and lower rows are utilized first). In addition, unlike YACR2 [13] which explicitly constructs the vertical constraint graph, vertical constraint violations (VCV) can be simply checked and avoided by using a simple heuristic method [3]. In Figure 9, we present the new PDTA algorithm. It works as follows: first, subnets are sorted in ascending order of their leftmost end-points, then an unprocessed track $T$ as per sequence of tracks (described 
before) is picked. The algorithm then places the horizontal segment of a subnet having the lowest left-end position if placing the net to the current track satisfies all constraints such as HC, VC and NC. Otherwise, the next subnet with the lowest left-end position is attempted. This process is repeated until all subnets are assigned to tracks. Unassigned subnets may exist in real fields because of channel area shortages or cyclic VCs. To solve those problems, we first attempt to place the unassigned subnets to empty spaces of processed tracks with considering only $\mathrm{HC}$ and NC. If the unassigned subnets still remain, then we should increase the channel area and add a new track to the center of the channel, and then repeat the above process.

After the PDTA step, to connect pins to the track we used the maze routing routines presented in YACR2. Due to space limitations, we do not describe the maze routing routines in this paper; please see [13] for details.

\section{EXTENSIONS}

When a M3 layer is available for routing, we have limitations as to how we can exploit M3 with the cell model (cf. Figure 4). More precisely, the pin layer is M1, which in turn means that a $\mathrm{HVH}$ routing scheme cannot be used. Therefore, when connecting to an M1-pin from an M3-track, this connection may have a conflict with neighboring M2 wires. As a result, to improve the three-layer routing efficiency the pin layer should be changed from M1 layer to M2 layer. In such a case, the power/ground pins inside the cell also must be changed from M2 layer to M1 or M3 layers. In addition, in order to use $\mathrm{HVH}$ routing scheme, the VC definition should be modified as described in [3]; if at any column, the net, which enters the channel from the bottom, was assigned to track $r$, and the net, which enters the channel from the top, was assigned track $r+1$, then this is also considered a vertical-constraint violation.

Although our algorithm can be applied to the three-layer routing with the considerations given above, the current implementation only addresses the two-layer routing problem.

\section{EXPERIMENTAL RESULTS}

TANAR is implemented in C++ on an Ultra-2 Sun Spark workstation, and tested on the Deutsch difficult problem [14] and five other randomly generated benchmark circuits. The characteristics of the channel routing problems are summarized in Table 2. The circuits are generated with 100 nets to 500 nets. We assume that in each benchmark circuit, the member count of $N_{C}, N_{S}, N_{T}$ and $N_{B}$ is $10 \%, 15 \%, 25 \%$ and $50 \%$ of the total nets, respectively. The percentages for each net type are considered based on the peripheral circuits. Nets are randomly assigned a type to meet the aforementioned distribution profile.

The maximum allowable peak noise $A N$ is specified for nets in $N_{C}$ or $N_{S}$, that is, the $25 \%$ of all nets in each

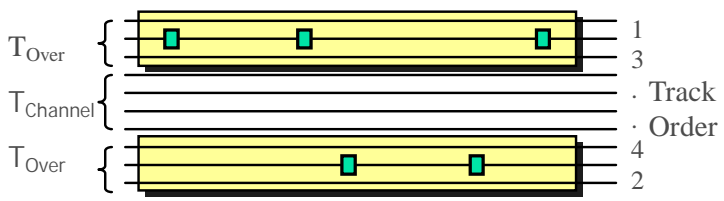

Figure 8. Track ordering

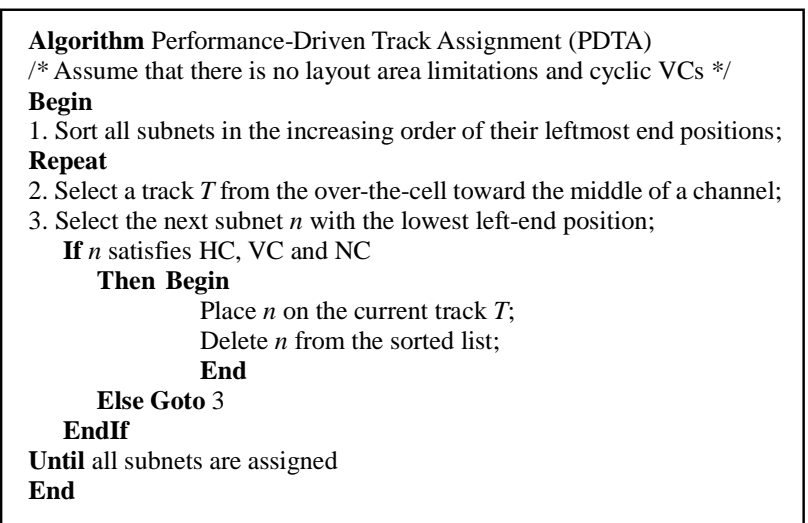

Figure 9. The performance-driven track assignment algorithm

benchmark circuit have the noise constraint, ranging from $10 \%$ to $30 \%$ of supply power voltage(we used uniformly distributed $A N$ values in that range). In addition, we randomly selected the critical-sink among sinks of nets in $N_{T}$.

We compared two channel routing flows, TANAR (PDNP+PDTA) and CONV (NP+TA), in terms of the peak crosstalk noise for $N_{C}$ and $N_{S}$, the critical-sink delay for $N_{T}$, and the number of tracks in the channel and over-the-cell. The conventional flow (CONV) consists of a MST-based partitioning step (NP) followed by a noise unaware track assignment step (TA). More precisely, the NP step partitions all nets into two-pin subnets using the MST topology in the same way as noise sensitive nets are partitioned by PDNP. TA used the same procedure as that employed in PDTA except it does not consider any noise constraints. As a result, the CONV flow tries to minimize the total routing length and the number of tracks used in the channel while satisfying the $\mathrm{HC}$ and VC.

TABLE 2. The characteristics of test examples

\begin{tabular}{|c|c|c|c|}
\hline Test Examples & \# of Nets & \# of Columns & Channel Density \\
\hline Deutsch & 72 & 174 & 19 \\
\hline Rand100 & 100 & 220 & 20 \\
\hline Rand200 & 200 & 480 & 39 \\
\hline Rand300 & 300 & 720 & 60 \\
\hline Rand400 & 400 & 870 & 81 \\
\hline Rand500 & 500 & 1200 & 90 \\
\hline
\end{tabular}

The results for the comparison are shown in Table 3 for test examples. Note that, compared to CONV, TANAR reduces the peak crosstalk noise for all sensitive nets by $40 \%$ or more for all test examples. The critical-sink delay from a source for timing-critical nets estimated using 
HSPICE with TSMC $0.18 \mu \mathrm{m} / 1.8 \mathrm{v}$ model: wire resistance $0.078 \Omega / \mu \mathrm{m}$, coupling capacitance $0.1 \mathrm{fF} / \mu \mathrm{m}$ and bottom capacitance $0.02 \mathrm{fF} / \mu \mathrm{m}$. Furthermore, we assumed that the source of each net is driven by an inverter $(\mathrm{Wp}=1.74 \mu \mathrm{m}$, $\mathrm{Wn}=1.2 \mu \mathrm{m}, \mathrm{Lp}=\mathrm{Ln}=0.18 \mu \mathrm{m})$, and each sink has $3.0 \mathrm{fF}$ for the load capacitance. TANAR improves upon CONV by reducing this delay from $4 \%$ to $16 \%$ (depending on the test case). In addition, TANAR reduces the number of tracks used inside channel by effectively using over-the-cell tracks. Note that the relative delay performance of TANAR improves as the benchmark size increases.

In summary, the experimental results demonstrate that the proposed fixed routing topology for each net according to its net type and performance constraints works effectively for the channel routing problem. As a result, the key issues in the interconnect design of peripheral circuits for memory devices have been addressed by the proposed algorithm.

\section{CONCLUSIONS}

We solved the automatic routing problem of memory peripheral circuits as an over-the-cell channel routing problem with a novel interconnect design technique. Our approach handles noise sensitive nets and timing critical nets at the same time while efficiently utilizing the over-the-cell area. Experiments showed that the proposed routing algorithm reduces the routing channel height and at the same time reduces the peak crosstalk noise for noise sensitive nets and the critical-sink delay for timing critical nets. To the best of our knowledge, this is the first work that simultaneously reduces the crosstalk noise, delay and area during the over-the-cell channel routing.

\section{References}

[1] S. Shibatani, T. Sadakane, H Nakao, M. Terai and K. Okazaki, "A CMOS cell generation system for two-dimensional transistor placement," Proc. IEEE Custom
Integrated Circuits Conf., pp. 325-328, 1998.

[2] D. Braun, J. Burns, S Davadas, H. Ma, K. Mayaram, F. Romeo and A. L. Sangiovanni-Vincentelli,, "Chameleon: a new multi-layer channel router," Proc. ACM/IEEE Design Automation Conference, pp. 495-502, 1986.

[3] U. Yoeli, "A robust channel router," IEEE Trans. Computer-Aided Design, vol.10, 1991.

[4] R. Gidwani and N.A. Sherwani, "MISER: An integrated three layer gridless channel router and," Proc. IEEE International Conf. Computer-Aided Design, pp. 698-703, 1990.

[5] W. C. Elmore, "The transient response of damped linear network with particular regard to wideband amplifiers," $J$. Applied Physics, vol. 19, pp 55-63, 1948.

[6] H. P. Tseng, L. Scheffer and C. Sechen, "Timing- and crosstalk-driven area routing," Proc. ACM/IEEE Design Automation Conference, pp. 378-382, 1998.

[7] A. Vittal, L. H. Chen, M. Marek-Sadowska, K.P Wang, and S. Yang, "Crosstalk in VLSI Interconnections," IEEE Trans. Computer-Aided Design, vol.18, pp. 1817-1824, 1999.

[8] D. A. Kirkpatrick and A. L. Sangiovanni-Vincentelli, "Digital sensitivity: Predicting signal interaction using functional analysis," Proc. IEEE International Conf. Computer-Aided Design, pp. 536-541, 1996.

[9] B. M. Goni and T. Arslan, An Evolutionary 3D Over-the-Cell Router, IEEE International ASIC/SOC Conference, pp. 206 - 209, 1999.

[10] I. Ali, S. Roy and G. Shinn, "Chemical-mechanical polishing of interlayer dielectric: A review," Solid State Technology, 37(10), pp. 63-70, 1994.

[11] A. Prim, "Shortest connecting networks and some generalizations," Bell System, Tech. J., vol. 36, pp. 1389-1401, 1957.

[12] K. D. Boese, A. B. Kahng, B. A. McCoy and G Robins, "Near-optimal critical sink routing tree constructions," IEEE Trans. Computer-Aided Design, vol.14, pp1417-1436, 1995.

[13] J. Redd, A. Sangiovanni-Vaincentelli, and M. Santomauro, "A new symbolic channel router: YACR2," IEEE Trans. Computer-Aided Design, vol. CAD-4, pp. 208-219, 1985.

[14] Burstein and R. Pelavin, "Hierarchical channel router," Integration VLSI J., vol. 1, pp. 21-38, 1983.

TABLE 3. Routing results comparison between TANAR and CONV

\begin{tabular}{|c|c|c|c|c|c|c|c|c|}
\hline \multirow{3}{*}{$\begin{array}{c}\text { Test } \\
\text { Examples }\end{array}$} & \multicolumn{4}{|c|}{ TANAR } & \multicolumn{4}{|c|}{ CONV } \\
\hline & \multirow{2}{*}{$\begin{array}{l}\text { Peak Noise }(\mathrm{V}) \\
\text { for } N s \text { and } N_{C}\end{array}$} & \multirow{2}{*}{$\begin{array}{c}\text { CS-Delay(ps) } \\
\text { for } N_{T}\end{array}$} & \multicolumn{2}{|c|}{ \# of Used Tracks } & \multirow{2}{*}{$\begin{array}{l}\text { Peak Noise }(\mathrm{V}) \\
\text { for } N s \text { and } N_{C}\end{array}$} & \multirow{2}{*}{$\begin{array}{l}\text { CS-Delay (ps) } \\
\text { for } N_{T}\end{array}$} & \multicolumn{2}{|c|}{ \# of Used Tracks } \\
\hline & & & $\begin{array}{l}\text { In-the- } \\
\text { Channel }\end{array}$ & $\begin{array}{l}\text { Over-the- } \\
\text { Cell }\end{array}$ & & & $\begin{array}{l}\text { In-the- } \\
\text { Channel }\end{array}$ & $\begin{array}{l}\text { Over-the- } \\
\text { Cell }\end{array}$ \\
\hline Deutsch & $0.17(67.0 \%)$ & $28.9(4.0 \%)$ & 13 & 24 & 0.53 & 30.4 & 16 & 15 \\
\hline Rand100 & $0.06(40.9 \%)$ & $26.5(5.6 \%)$ & 16 & 27 & 0.10 & 28.1 & 17 & 14 \\
\hline Rand200 & $0.20(67.7 \%)$ & $33.8(7.3 \%)$ & 25 & 45 & 0.62 & 36.5 & 26 & 28 \\
\hline Rand300 & $0.36(60.0 \%)$ & $36.9(7.7 \%)$ & 44 & 77 & 0.90 & 40.0 & 46 & 40 \\
\hline Rand400 & $0.41(62.0 \%)$ & $40.7(13.5 \%)$ & 41 & 86 & 1.08 & 47.1 & 45 & 47 \\
\hline Rand500 & $0.52(57.4 \%)$ & $46.4(16.5 \%)$ & 54 & 92 & 1.22 & 55.6 & 56 & 56 \\
\hline
\end{tabular}

